\title{
The Structure and Composition of Deciduous Enamel Affected by Local Hypoplastic Autosomal Dominant Amelogenesis Imperfecta Resulting from an ENAM Mutation
}

\author{
R.C. Shore ${ }^{a} \quad$ B. Bäckman ${ }^{d} \quad$ C. Elcock ${ }^{b} \quad$ A.H. Brook ${ }^{c} \quad$ S.J. Brookes ${ }^{a} \quad$ J. Kirkham ${ }^{a}$ \\ a Leeds Dental Institute, University of Leeds, Leeds, ${ }^{b}$ School of Clinical Dentistry, University of Sheffield, Sheffield, \\ and 'SChool of Dental Sciences, University of Liverpool, Liverpool, UK; d Department of Odontology/Pedodontics, \\ University of Umeå, Umeå, Sweden
}

\section{Key Words}

Enamel $\cdot$ Amelogenesis imperfecta $\cdot$ Local hypoplastic •

ENAM $\cdot$ Enamelin

\begin{abstract}
In a group of families in northern Sweden, a mutation in the ENAM gene (predicted to produce a highly truncated protein) results in the local hypoplastic form of autosomal dominant amelogenesis imperfecta. In this study, sections of deciduous teeth from members of 3 of these families were examined by scanning electron microscopy (SEM) and the enamel mineral was analysed by energy dispersive $\mathrm{X}$-ray spectroscopy (EDX). The sections were also probed with antibodies raised to a conserved sequence of the enamelin protein. Selected intact teeth were first analysed by digital imaging and ascribed with an 'Enamel Defects Index' (EDI) score. SEM of tooth sections revealed disrupted prism morphology and the prisms had a glass-like appearance in some areas. These areas of dysplasia were sometimes irregular but formed regular arrays in others. Comparison of EDI scores with SEM indicated that in one tooth the surface had no measurable defects but significant defects were present in the underlying enamel microstructure. SEM immunohistochemistry with the antibody raised to a fragment of the enamelin protein produced positive, but light, labelling throughout normal enamel. In dysplastic areas, however, the
\end{abstract}

labelling intensity appeared to be reduced. The results indicate that the presence of functional enamelin in the correct amounts is necessary for correct prism morphogenesis. In addition, a combination of EDI and structural analysis indicate that defects in enamel microstructure are not necessarily visible as defects on the surface of the tooth, suggesting the possibility, at least, that some instances of under-diagnosis may occur.

Copyright $\odot 2009$ S. Karger AG, Basel

\section{Introduction}

Both the local and smooth hypoplastic forms of autosomal dominant amelogenesis imperfecta (AI) are hereditary conditions for which the gene defect was mapped to the long arm of chromosome 4, where the enamelin gene (ENAM) emerged as the most likely candidate. Subse-

\begin{tabular}{ll} 
Abbreviations used in this paper \\
\hline AI & amelogenesis imperfecta \\
EDI & Enamel Defects Index \\
EDX & energy dispersive X-ray spectroscopy \\
ENAM & enamelin gene \\
SEM & scanning electron microscopy
\end{tabular}


quently, specific mutations in this gene were described which resulted in either smooth or local hypoplastic AI [Rajpar et al., 2001; Kida et al., 2002; Mårdh et al., 2002; Hart et al., 2003; Gutierrez et al., 2007; Pavlic et al., 2007]. The clinical appearance of the local hypoplastic form varies from rows of pits to grooves to large single hypoplastic areas. The enamel in these areas appears to be of normal hardness on probing. This clinical phenotype varies between families and even between members of the same family. In one group of families in northern Sweden the defect in ENAM was found to be a nonsense mutation in exon 5 which resulted in a much truncated expressed protein [Mårdh et al., 2002]. There is some data on the underlying structure of the enamel affected by the local hypoplastic form [Pavlic et al., 2007], where there appeared to be a loss of prism definition. There is some earlier data on the smooth generalised hypoplastic form, however, where several teeth from one affected individual were analysed [Wright et al., 1991]. Within these teeth, there appeared to be disruption in the alignment of the hydroxyapatite crystals and a concomitant loss of normal prism structure and definition (as in the local hypoplastic form). A later paper also suggested that the affected enamel retained a relatively high protein content [Wright et al., 1992]. More recently, the microscopic phenotype arising from 2 specific ENAM mutations, which resulted in generalised smooth hypoplasia, was published [Wright et al., 2009]. The aim of the current study was to characterise the structure and composition of a number of teeth from individuals from several families where the teeth demonstrate a local hypoplastic phenotype and where the precise mutation in the ENAM gene is known. This would then, in turn, allow a better understanding of the features underlying this clinical defect and help throw light on the role of enamelin in the formation of normal enamel architecture. An additional aim was to investigate the possibility that structural defects in the interior of the enamel might not necessarily be reflected in clinically visible surface defects or variations in enamel thickness.

\section{Materials and Methods}

\section{Families}

Exfoliated deciduous teeth were collected from members of 3 families in northern Sweden with the local hypoplastic clinical phenotype and an autosomal dominant inheritance pattern. Teeth with visible signs of caries were discarded and 11 teeth were selected for study. These were families ' 7 ' ( 2 individuals, 6 teeth), ' 9 ' ( 1 individual, 2 teeth) and ' $W$ ' ( 1 individual, 3 teeth) published previously by Bäckman and Holmgren [1988] and more recently by Mårdh et al. [2002], who reported that the underlying gene defect common to all 3 families was a nonsense mutation in the enamelin gene, predicted to produce a much truncated protein (g.2382A > T, p.K53X). All individuals from whom these teeth were collected were heterozygous with respect to ENAM [Mårdh et al., 2002]. Type-matched control teeth were obtained by donation, with informed consent, via the paediatric clinic of the Leeds Dental Institute.

\section{EDI and Image Analysis}

Before processing, the EDI value was determined for the labial/ buccal and lingual surfaces of all teeth from one individual (chosen at random from one of the families) by the method reported by Brook et al. [2001] and Elcock et al. [2006], employing visual inspection and using image analysis of digital images. The index is binary in nature and has a value range from $0,0,0$ to $1,1,1$, the former indicating no visible defect of any kind (hypoplasia, opacity or post-eruptive breakdown) is present and the latter indicating all 3 are present on the tooth surface under scrutiny. The teeth used in this study are a subset of a much larger collection of teeth for which the EDI has been determined [Smith et al., 2008].

\section{Tooth Section Preparation}

All teeth were sectioned longitudinally in a labio-lingual direction using an Accutom-5 cutter with a peripheral diamond cutting disc and minimal water cooling to minimise loss of any water soluble components within the enamel. Three sections in the midline were polished plano-parallel with 1,200 grade carborundum paper to a thickness of $100 \mu \mathrm{m}$, then acid-etched for exactly $15 \mathrm{~s}$ with $35 \%$ phosphoric acid to remove the smear layer.

Scanning Electron Microscopy and Elemental Analysis (EDX)

One section from each tooth was mounted on an aluminium stub and sputter-coated with gold. Gold was used in preference to the more traditional coating with carbon for elemental analysis in order that any endogenous carbon content could be more accurately determined. Microstructural analysis was undertaken using a Jeol 35 scanning electron microscopy (SEM) fitted with the Deben 'Genie' upgrade (Deben Engineering, Debenham, UK). Elemental analysis was performed using a detector fitted with an ultra-thin window and driven by WinEDS 3 software (Thomson Scientific, Carlton, Vic., Australia). The EDX detector was calibrated (for peak position and relative peak heights) using powdered apatite and 'Analar' grade $\mathrm{CaCO}_{3}$ and $\mathrm{Ca}\left(\mathrm{NO}_{3}\right)_{2}$ pressed into $8-\mathrm{mm}$ diameter $\times 2-\mathrm{mm}$ discs using a pill press at 8 tons. These discs were mounted and sputter-coated with gold in the same way as the sections. All analyses were performed under the same operating conditions of aperture size, magnification and beam current. Each section was divided into 5 zones around the crown: cervical half (zone ' $i$ ') and incisal half (zone 'ii') of the labial aspect of the tooth; cervical and incisal halves of the lingual aspect (zones 'iv' and ' $v$ '); and the area immediately around the incisal edge or cusp (zone 'iii'). In each of the 5 zones, a detailed analysis of the prism architecture was undertaken. In addition, in each zone, 5 EDX spectra were obtained in a line running from the amelodentinal junction to the enamel surface. Spectra were also obtained from dentine adjacent to the areas of affected enamel where the tissue had a similar degree of mineralisation but where there was a high protein (collagen) content. 
Fig. 1. Enamelin (human): residues $1-420$ [Hu et al., 2000] The antibody was raised to synthesised peptide shown as highlighted. * Signal peptide; ${ }^{*} 13$-residue truncated secreted protein.
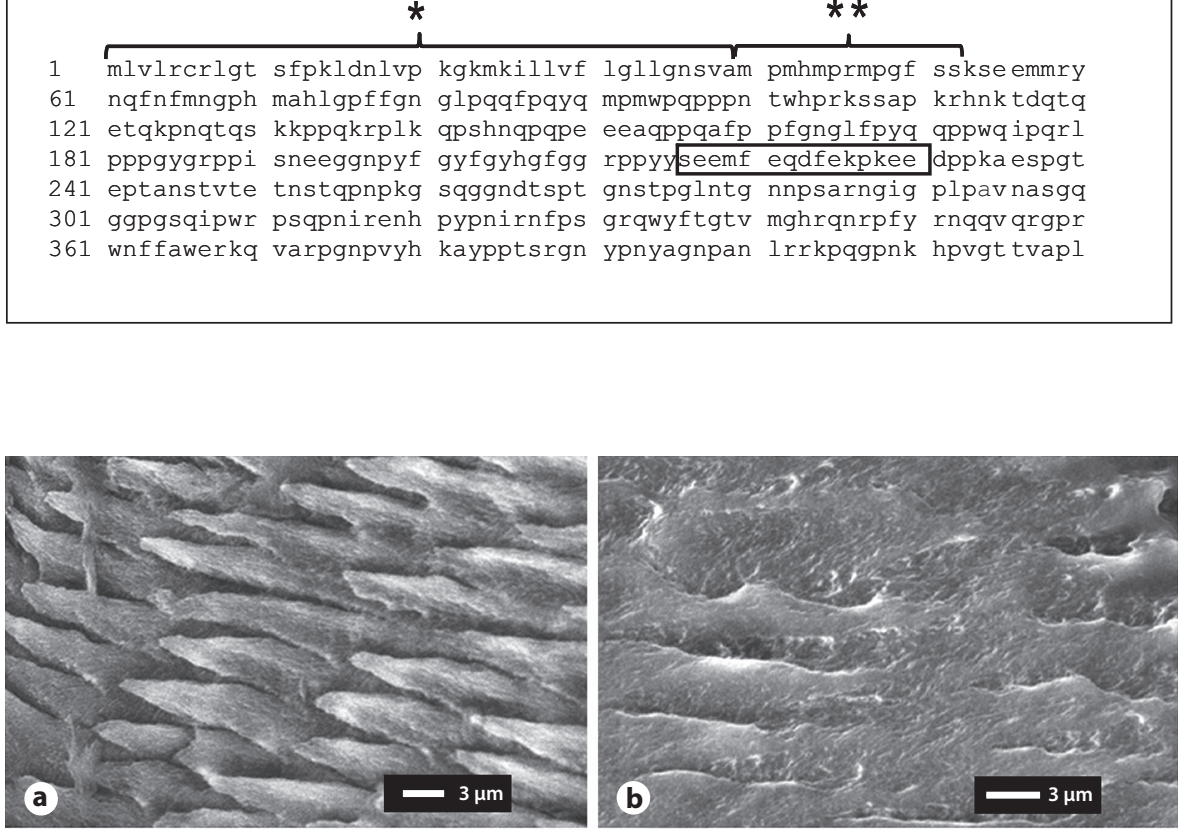

Fig. 2. Scanning electron micrographs of longitudinal sections of a control tooth (upper central incisor, zone 'ii') (a) and dysplastic areas of affected teeth (b-d). Lower lateral incisor, zone 'i' (b), lower central incisor, zone 'ii' (c), upper central incisor, zone 'iii' (d). Note the lack of visible prisms and the highly disordered nature of the crystals in c and the glass-like appearance of the prisms in $\mathbf{d}$.
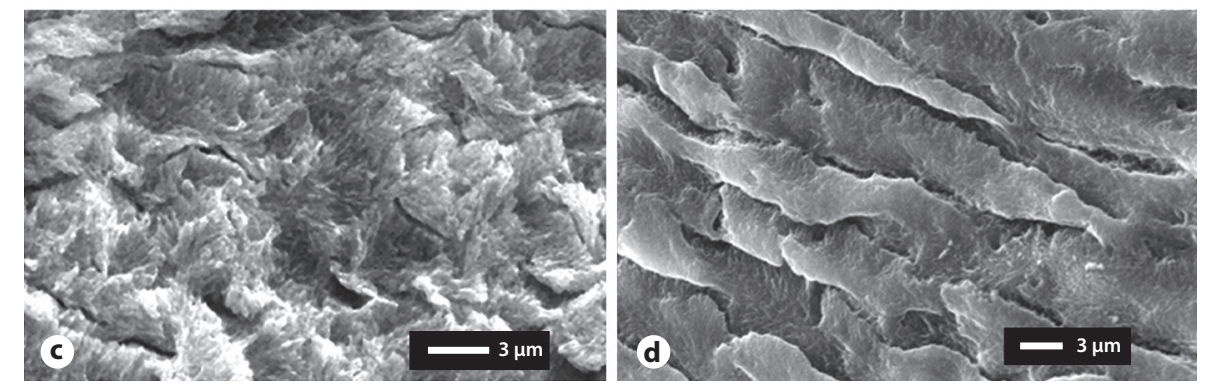

\section{SEM Immunohistochemistry}

A 2 nd section from each tooth was reacted with a polyclonal antibody raised to a synthetic peptide representing 12 residues of the human enamelin molecule (fig. 1) [Hu et al., 2000], followed by a 2 nd antibody conjugated to $1-\mu \mathrm{m}$ polystyrene spheres, then washed, dried, sputter-coated and viewed by SEM. The facing surface of the 3rd section acted as an immunological control with a non-relevant primary antibody [Shore et al., 2000]. The 12 residues chosen for the synthetic peptide fall outside the 13-residue truncated protein coded for by the mutant ENAM.

\section{Results}

\section{SEM}

Areas within the AI enamel were characterised by an irregular arrangement of the constituent crystals within the prisms when compared with enamel from similar sites in the teeth of unaffected individuals (fig. 2a), such that prism boundaries were difficult to distinguish when one prism combined with adjacent prisms (fig. 2b). In regions which were apparently more affected, the prism structure was essentially completely obscured (fig. 2c). Often the prisms, when present, had a 'glass-like' appearance where individual crystals seemed to have fused (fig. 2d). The severity and frequency of these structural abnormalities varied between siblings within the same family and between different teeth in the same individual. Severity also varied between different regions of the same tooth. Significantly, one of the teeth for which the EDI was determined returned a score of $0,0,0$ (i.e., no visible external defect on any surface; fig. $3 a$ ), and yet subsequent detailed structural analysis of this tooth indicated microstructural defects beneath these apparently normal surfaces (fig. 3b). Clinically, therefore, this tooth would have been classified as unaffected. In addition, several teeth (to a greater or lesser degree) appeared to 
Fig. 3. Photograph of the labial surface of an upper central incisor scoring $0,0,0$ on the EDI (a) and a corresponding SEM of the enamel beneath the surface of zone 'ii' of this tooth, approximately $100 \mu \mathrm{m}$ from the amelodentinal junction (b). Note the disordered prisms, similar to those in fig. 2b. c SEM of a longitudinal section of zone 'ii' of an affected tooth showing a highly ordered arrangement of the affected areas (arrows indicate borders between a region of ordered prisms and 2 regions of highly disordered prisms).

Fig. 4. Scanning electron micrographs of enamel reacted with an anti-enamelin peptide antibody and 2nd antibody conjugated to $1-\mu \mathrm{m}$ spheres. a Enamel from an unaffected tooth showing spheres distributed relatively uniformly around the prisms. b Increased density of labelling (arrowed) adjacent to the amelodentinal junction (arrowhead). c Affected area of an affected tooth. Note the reduced labelling around the prisms and the tendency to 'clump'. d A non-relevant primary antibody and the same 2nd antibody used in a-c. Note the lack of labelling around the prisms, suggesting the 'prism boundary' location of the labelling in $\mathbf{a}$ is not the result of an 'edge' artefact.
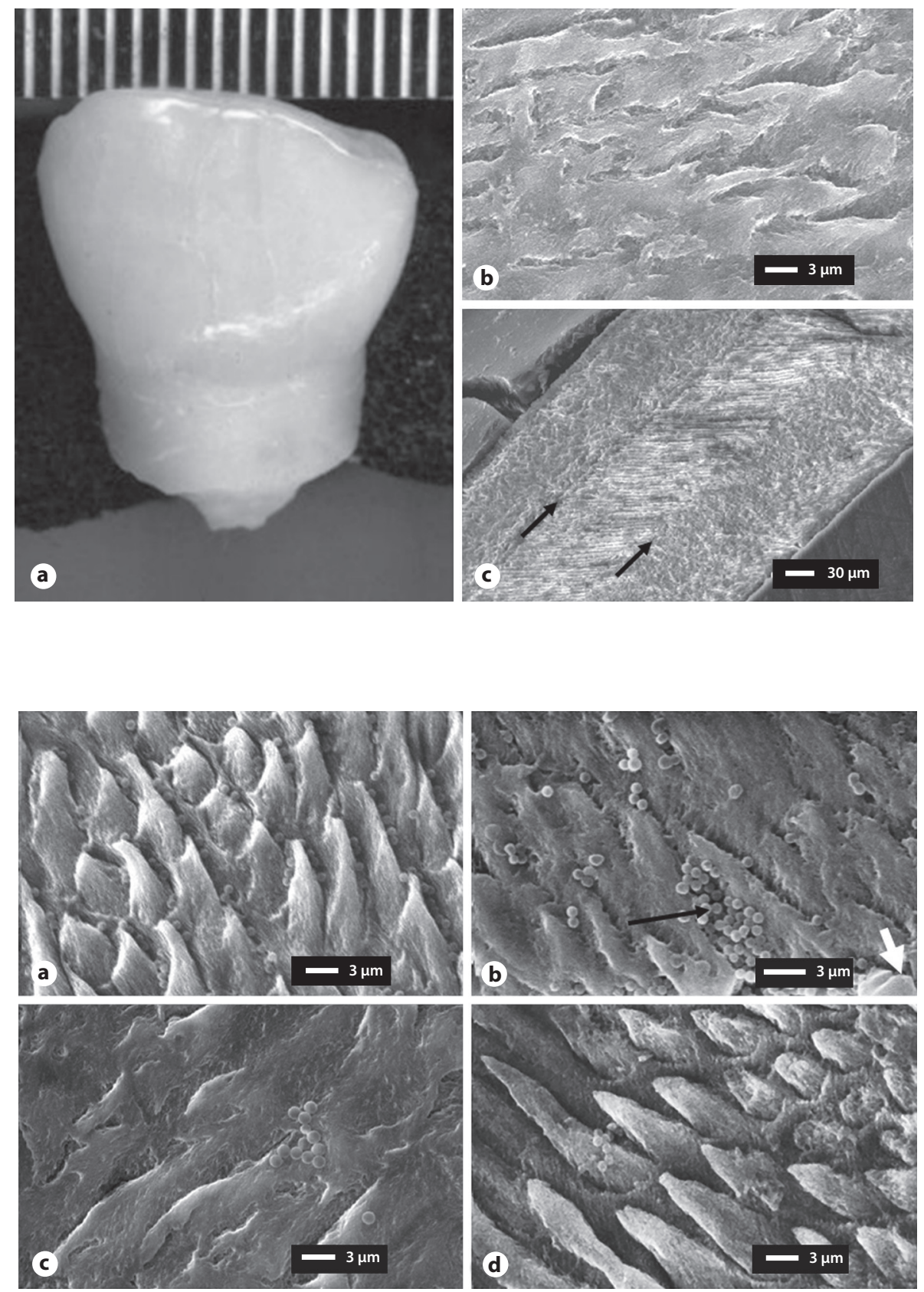

have an extremely regular arrangement of the dysplastic enamel (fig. 3c), with a band of relatively normal prisms between inner and outer regions of highly abnormal ones. The boundary between the two was well demarcated.

\section{SEM Immunohistochemistry}

In control (unaffected) teeth, diffuse labelling with the anti-enamelin antibody was evident throughout the enamel (fig. 4a), with a noticeably increased labelling in- tensity adjacent to the amelodentinal junction (fig. $4 \mathrm{~b}$ ). In regions of affected teeth which appeared structurally normal or where only mild prism dysplasia was present, similar levels of labelling were evident. In severely dysplastic areas, however, there appeared to be, on the basis of visual inspection, a much reduced labelling intensity (fig. 4c), with the reduced number of spheres sometimes being more 'clumped' and less diffuse. 


\section{Elemental Analysis (EDX)}

The analysis of the data showed that the enamel mineral composition from both affected and unaffected regions in the affected teeth was indistinguishable from type-matched controls, with Ca:P molar ratios of 1.551.70 .

\section{Discussion}

In the group of families studied here, where the clinical presentation is autosomal dominant local hypoplastic AI and the gene defect is likely to result in the secretion, if at all, of a highly truncated enamelin molecule, the consistent underlying microstructural defect is in varying degrees of prism disarrangement. This results in irregular prisms which often form anastamoses and in severe cases the prism outline may be lost completely, a phenotype reported for other ENAM mutations [Pavlic et al., 2007; Wright et al., 2009]. An additional feature which may be superimposed on these dysplastic prisms is the lack of definition of individual crystals, giving the prisms a glasslike appearance. On the basis of the elemental analysis, however, the mineral itself making up these crystals appears to differ little from that of control (unaffected) teeth. Perhaps more significant, however, is the presence of a dysplastic prism structure beneath a clinically normal enamel surface (i.e., one scoring $0,0,0$ on the EDI) which could potentially result in whole dentitions being clinically classified as 'unaffected', but where major structural defects could be present in the underlying enamel. This may then have consequences for the inheritance pattern ascribed to the family from which the individuals is taken. Another instance where there may be a lack of relationship between overt hypoplasia and subsurface defects is seen in the heterozygote enamelin knockout mouse [ $\mathrm{Hu}$ et al., 2008]. In these animals the maxillary (although, interestingly, not the mandibular) incisors of the $\mathrm{Enam}^{+/-}$mice appeared outwardly normal but demonstrated a variable decrease in the subsurface pigment. Indeed that study highlighted a number of phenotypic differences between the mandibular and maxillary teeth. Whilst the mandibular teeth consistently demonstrated a dose response between $\mathrm{Enam}^{+/+}, \mathrm{Enam}^{+/-}$and Enam ${ }^{-/-}$in terms of mineral to protein ratios and mineral percentage, the $\mathrm{Enam}^{+/+}$and $\mathrm{Enam}^{+/-}$were indistinguishable in the maxillary teeth.

The sometimes very regular arrangement of the areas of abnormal prisms in the longitudinal sections used in this present study is interesting. It possibly suggests that groups of ameloblasts are producing abnormal prism ar- chitecture while adjacent groups are not (or at least to a lesser extent), resulting in a sometimes 'striped' appearance in the enamel. There is no immediate explanation as to how groups of ameloblasts which possess the same ENAM mutation produce enamel with apparently different characteristics both in terms of enamel thickness and/ or prism and crystal morphology. If this was an X-linked condition rather than autosomal, then Lyonisation might offer an explanation. As it is indeed autosomal, then some other explanation must be sought. This might possibly be in terms of some other modifying factor or factors that may mitigate the effects of the truncated protein to varying degrees in certain cohorts of cells. All individuals from which these teeth were collected were heterozygous with respect to ENAM [Mårdh et al., 2002], thus essentially representing a human equivalent of the $E N A M^{+/-}$ mouse. Enam mutations which, as is likely in this case, cause haploinsufficiency have been shown to result in more localised rather than generalised hypoplasia.

The results of the SEM immunohistochemistry with an antibody raised to a peptide corresponding to residues 216-230 of human enamelin produced diffuse labelling in normal enamel suggesting that small amounts of enamelin or enamelin fragments remain in the mature tissue. Because of the nature of the labelling used (the $1-\mu \mathrm{m}$ spheres), it is impossible to say whether this is primarily in prism cores or prism sheaths. The highest labelling intensities, however, were found along, and radiating from, the amelodentinal junction, possibly corresponding to the histological structures referred to as 'enamel tufts' and constituting part of the so-called 'tuft protein'. Previous work on developing pig teeth had already suggested an accumulation of enamelin or enamelin fragments along the amelodentinal junction [Uchida et al., 1991]. The reduced labelling intensity in the affected areas of AI enamel could arise from a slight decrease in the expression of the normal, full length protein in that cohort of cells, such that the amount of protein secreted into the tissue is decreased and therefore the amount of residual protein remaining in the mature tissue falls below the detection limit of this detection method. This might imply that the presence of the normal protein, at a level sufficient to leave a residue that can be detected, is necessary for correct prism and crystal formation.

\section{Acknowledgements}

This work was generously supported by the Wellcome Trust (Programme 075945/Z/04/Z). We are grateful to Mrs. Jackie Hudson and Dr. Sarah Myers for technical assistance. 


\section{References}

Bäckman, B., G. Holmgren (1988) Amelogenesis imperfecta: a genetic study. Hum Hered 38: 189-206.

Brook, A.H., C. Elcock, A.-L. Hallonsten, S. Poulsen, J. Andreasen, G. Koch, C.A. Yeung, T. Dosanjh (2001) The development of a new index to measure enamel defects; in Brook, A.H. (ed): Dental Morphology. Sheffield, Sheffield Academic Press, pp 59-66.

Elcock, C., D.L. Lath, J.D. Luty, M.G. Gallagher, A. Abdellatif, B. Bäckman, A.H. Brook (2006) The new Enamel Defects Index: testing and expansion. Eur J Oral Sci 114(Suppl 1): 35-58, discussion 39-41, 379.

Gutierrez, S.J., M. Chaves, D.M. Torres, I. Briceno (2007) Identification of a novel mutation in the enamalin gene in a family with autosomal-dominant amelogenesis imperfecta. Arch Oral Biol 52: 503-506.

-Hart, T.C., P.S. Hart, M.C. Gorry, M.D. Michalec, O.H. Ryu, C. Uygur, D. Ozdemir, S. Firatli, G. Aren, E. Firatli (2003) Novel ENAM mutation responsible for autosomal recessive amelogenesis imperfecta and localised enamel defects. J Med Genet 40: 900 906.

Hu, C.C., T.C. Hart, B.R. Dupont, J.J. Chen, X. Sun, Q. Qian, C.H. Zhang, H. Jiang, V.L. Mattern, J.T. Wright, J.P. Simmer (2000) Cloning human enamelin cDNA, chromosomal localization, and analysis of expression during tooth development. J Dent Res 79: 912-919.
Hu, J.C., Hu, Y., C.E. Smith, M.D. McKee, J.T. Wright, Y. Yamakoshi, P. Papagerakis, G.K. Hunter, J.Q. Feng, F. Yamakoshi, J.P. Simmer (2008) Enamel defects and ameloblast-specific expression in Enam knock-out/lacz knock-in mice. J Biol Chem 283: 1085810871.

Kida, M., T. Ariga, T. Shirakawa, H. Oguchi, Y. Sakiyama (2002) Autosomal-dominant hypoplastic form of amelogenesis imperfecta caused by an enamelin gene mutation at the exon-intron boundary. J Dent Res 81: 738742.

Mårdh, C.K., B. Bäckman, G. Holmgren, J.C. Hu, J.P. Simmer, K. Forsman-Semb (2002) A nonsense mutation in the enamelin gene causes local hypoplastic autosomal dominant amelogenesis imperfecta (AIH2). Hum Mol Genet 11: 1069-1074

Pavlic, A., M. Petelin, T. Battelino (2007) Phenotype and enamel ultrastructure characteristics in patients with ENAM gene mutations g.13185-13186insAG and 8344delG. Arch Oral Biol 52: 209-217.

Rajpar, M.H., K. Harley, C. Laing, R.M. Davies, M.J. Dixon (2001) Mutation of the gene encoding the enamel-specific protein, enamelin, causes autosomal-dominant amelogenesis imperfecta. Hum Mol Genet 10: 1673-1677.
Shore, R.C., J. Kirkham, S.J. Brookes, S.R. Wood, C. Robinson (2000) Distribution of exogenous proteins in caries lesions in relation to the pattern of demineralisation. Caries Res 34: 188-193.

Smith, R.N., C. Elcock, A. Abdellatif, B. Bäckman, J.M. Russell, A.H. Brook (2008) Enamel defects in extracted and exfoliated teeth from patients with Amelogenesis Imperfecta, measured using the extended enamel defects index and image analysis. Arch Oral Biol. doi:10.1016/j.archoralbio.2008.07.008

Uchida, T., T. Tanabe, M. Fukae, M. Shimizu (1991) Immunocytochemical and immunochemical detection of a $32 \mathrm{kDa}$ nonamelogenin and related proteins in porcine tooth germs. Arch Histol Cytol 54: 527-538.

Wright, J.T., T.C. Hart, P.S. Hart, D. Simmons, C. Suggs, B. Daley, J. Simmer, J. Hu, J.D. Bartlett, Y. Li, Z.A. Yuan, W.K. Seow, C.W. Gibson (2009) Human and mouse enamel phenotypes resulting from mutation or altered expression of AMEL, ENAM, MMP20 and KLK4. Cells Tissues Organs 189: 224-229.

-Wright, J.T., C. Robinson, J. Kirkham (1992) Enamel protein in smooth hypoplastic amelogenesis imperfecta. Pediatr Dent 14: 331 337.

Wright, J.T., C. Robinson, R. Shore (1991) Characterization of the enamel ultrastructure and mineral content in hypoplastic amelogenesis imperfecta. Oral Surg Oral Med Oral Pathol 72: 594-601. 